\title{
Un Criterio Ideal: Reflexiones para la Educación Turística
}

\begin{abstract}
Pablo Suazo Villalón ${ }^{1}$
RESUMEN: Expresa ciertas observaciones sobre como se aborda el turismo y como puede mejorar la comprensión integral en tres aspectos relevantes: profesión, actividad y disciplina, los cuales deberían potenciarse en la educación turística a través de un criterio fundamentado en la conjunción educación-investigación y en la exploración hacianuevas perspectivas de pensamiento en la formación del estudiante de turismo.
\end{abstract}

PALABRAS CLAVES: criterio ideal, criterio inconexo, educación turística, investigación turística.

ABSTRACT: The article presents some observation on how tourism is dealt integrated comprehension of thee relevant aspects: profession, activity, and discipline. These aspects should be improved within tourism education through a criterion based on the educaction-research conjunction and the exploration ofnew thoyght tendencies in the information of tourism students.

KEYWORDS: ideal criterion, unconnected criterion, tourist education, tourist research.

\section{Introducción}

No cabe duda que el turismo es una alternativa tentadora. Laboralmente por las particularidades que significa trabajar en lugares donde otros descansan, por la posibilidad de interactuar con distintos tipos de personas y por ser una opción de empleo. Como disciplina de estudio el turismo es una interesante área y un campo

\footnotetext{
1. Administrador Turístico, licenciado en Turismo, Universidad de La Serena, Chile. Diplomado en calidad y Productividad de los Servícios Universidad Técnica Federico Santa María, Chile.

End.: Miguel Aguirre Perry 1920. Depto. 108, Fono: (56) (51) 214060 La Serena, Chile

E-mail: pablosuazo@yahoo.com
} 
nuevo de conocimiento sin embargo, estos argumentos se vuelven sensibles a la hora de observar el lento proceso de consolidación profesional del sector y el escaso reconocimiento académico del turismo. Esto parece repercutir en el desarrollo de la disciplina y de la actividad turística. Por consiguiente debiésemos reflexionar sobre estos temas no sin antes señalar algunas consideraciones pertinentes.

En este contexto podríamos decir que existe una cierta falta de conciencia colectiva sobre la verdadera importancia de la actividad y los estudios turísticos. Esto se observa cuando se considera al turismo como un fenómeno netamente económico al no involucrar las dimensiones social cultural y ambiental (Lickonish, 1994). Esto se manifiesta al momento de observar el comportamiento de algunos empresarios que operan en el medio, los cuales esperan retornos de utilidades en forma inmediata (Yasigi, 1998) carentes de visiones integrales.

Por otra parte, los representantes del sector público que consideran al turismo sólo en el tiempo que otros sectores productivos entran en crisis, lo que denota un cierto desconocimiento al instante de tomar decisiones frente al desarrollo turístico, factor que puede apreciarse en el divorcio entre los encargados de los organismos públicos del turismo con sus equipos técnicos, y porque no decir con el área en general (Cárdenas, 1986).

En el ámbito universitario estos antecedentes son tratados con una actitud pasiva identificándolos como factores exógenos a la acción de los estudiantes y académicos, lo que de alguna manera evidencia la falta de respuestas de los centros de estudios frente al desarrollo de la actividad y la disciplina, lo cual exhibe algunas deficiencias formativas en materia de comprender el sistema turístico y anticipar sus cambios (López, 1993).

Sin duda, dicha deficiencia podría ser argumento para que los otros actores de la actividad como son los empresarios y el sector público, apunten sus interrogantes sobre las habilidades y competencias de los profesionales del turismo, lo que de algún modo contribuiría a la visión netamente técnica, a la indefinición del rol profesional, y por último a una explicación del turismo como una actividad económica de resultados inmediatos.

Ante este panorama y en busca de análisis más estructurados hemos encaminado la reflexión sobre la base de una breve revisión bibliográfica y de apreciaciones formuladas en diversos coloquios formales e informales en el ámbito universitario.

\section{Distintos Criterios para Enfrentar el Turismo}

Al tenor de las consideraciones expuestas podríamos preguntarnos ¿Cómo se aborda el turismo? En otras palabras se interpreta dicha interrogante en el cómo se concibe la actividad y los estudios del fenómeno turístico por los involucrados directos en el desarrollo de éste; en especial desde la educación universitaria del turismo.
A nuestro entender existirian dos "Criterios" para abordar el turismo, uno que lo identificaremos como "Inconexo" y el segundo como "Ideal" (Fig. 1).

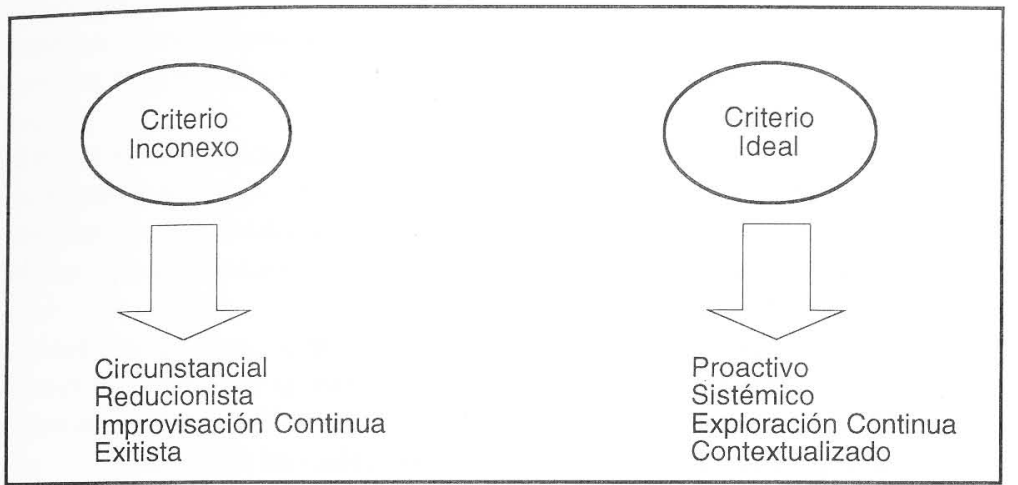

FIGURA 1- CARACTERISTICAS DE LOS CRITERIOS

El criterio Inconexo es el que no ha permitido establecer aproximaciones más exactas del funcionamiento y proyección de la actividad, debido a la escasa complementariedad entre los empresarios, gobierno, profesionales y centros de estudios, es justamente este argumento quien entrega el nombre al criterio. Este a su vez, tampoco ha dejado entender de manera sistémica el turismo limitando la comprensión del fenómeno desde visiones reducionista, un ejemplo categórico es cuando explicamos el turismo en función de una demanda o de la prestación del servicio. Ello ha provocado en ocasiones que las actividades, proyección e importancia de los estudios turísticos sean justificadas apelando a simples y burdas apariencias (Molina \& Rodríguez, 1991).

En otro sentido este tipo de enfoque circunstancial ha impedido dar respuestas e interpretaciones coherentes del desarrollo de la actividad, lo que se ha caracterizado por un largo peregrinar de improvisación continua frente a las diversas reacciones del sistema turístico impidiendo entregar aportes más sólidos a conocimiento científico del turismo, debido a la superficialidad de ciertos análisis que este criterio impone. Estos aspectos no sólo se observan al nivel de empresarios y gobierno, sino también se reflejan en la educación turística. En atención a 1 anterior la educación turística debería diseñar desde la analogía académico estudiantil nuevas perspectivas más integrales e innovadoras para el estudio del turismo, después de todo la educación turística tiene una mayor responsabilidad de dinamizar los cambios y el entendimiento del sistema sobre la base de la crítica y la reflexión.

La educación universitaria en turismo debe proporcionar un conjunto de "herramientas" dirigidas a la interpretación y la evolución de nuevos conocimientos, posibilitando al alumno desarrollar su capacidad crítica (Ansarah, 1998, p. 57). 
En adhesión a esta línea de pensamiento se suscribe el criterio ideal que debiese conducirnos a la comprensión sistémica del fenómeno. El criterio ideal a su vez contribuiría a establecer en forma más precisas las interrelaciones producidas entre los diferentes factores que evolucionan dinámicamente en el turismo (OMT, 1998). Esto podría alimentar la búsqueda de una nueva definición y comprensión más refinadas de él (Jafari, 1992).

En esta perspectiva, el desarrollo de la disciplina turística no puede estar sujeto a las particularidades que se presentan, pues debiese trabajar en forma proactiva, sistémica y orientadora, anticipando los escenarios que plantea el devenir de la actividad. Dichos escenarios necesitan ser vislumbrados, explicados y comprobados desde el accionar de la disciplina.

Hacia esta posición se dirige el criterio ideal, que se expresa como un discernimiento organizado e incorporado al proceso de formación universitaria del turismo. Ello permitiría colaborar al desarrollo científico, al mejoramiento de la actividad y al posesionamiento de los profesionales del área.

La construcción de criterios ideales para enfrentar la actividad turística lleva en sí un compuesto colectivo generado y sustentado en criterios individuales, alimentados por las divergencias naturales y espontáneas de mentes inquietas por e estudio del turismo, pero también por las experiencias y motivación de los más avezados, el cuerpo académico.

De la conjunción de los formadores venidos de otras disciplinas y el interés de nuevos investigadores adiestrados en las universidades donde se imparten carreras del ámbito, debieran surgir respuestas sólidas por medio del estudio sistemático del fenómeno. Tales respuestas podrán permitir aproximaciones perfeccionadas sobre la comprensión de las estructuras y sus funciones subyacentes del sistema turístico. Lo que se ha denominado "plataforma basada en el conocimiento, proceso que en otras latitudes, ya se ha iniciado" (Jafari, 1992).

\section{Del Criterio Ideal y la Condición Cambio}

El sustento del criterio ideal está dado por una mejor atingencia entre educación e investigación, lo que evidentemente es válido para toda ocupación científica. Dicha relación establece un flujo continuo de conocimiento provocando nuevos estudios, cuestionando los existentes, confirmando o consolidándolos, y al mismo tiempo ampliando su área de aplicación hacia nuevas dimensiones (Rejowski, 1998).

No obstante, es difícil realizar esta tarea por las cualidades del incipiente estado de maduración de la disciplina del turismo que no logra al parecer, alcanzar la consolidación académica como se supondría (López Palomeque et al., 1997).

Condiciones que podrían contribuir a esta situación son que gran parte de las investigaciones en turismo han carecido de bases metodológicas y/o teóricas, acompañadas de escasas críticas a su elaboración, esto ha impedido evaluar repercusiones en el sistema y consolidar la disciplina en cimientos científicos (Mathieson y Wall, 1990; OMT, 1995). Además existirían otras agravantes como la incorporación de personas que desconocen la estructura elemental de la investigación científica y que están incorporadas al desarrollo académico del turismo; la falta de interés por investigar de los estudiantes y profesores de las carreras de turismo o simplemente la ausencia de apetito por la generación de investigación (Boullón, 1988; OMT, 1995). Otro aspecto importante y digno de un análisis mayor es el alejamiento del concepto tradicional de universidad, en donde se ha privilegiado la docencia más que buscar el equilibrio óptimo entre docencia e investigación.

Por otraparte, resulta muchas veces innegables la desconexión entre educación e investigación en turismo, si se compara con otros sectores productivos (Ansarah, 1998). Cabe señalar que las entidades de educación que dictan carreras en el área parecen estar lejos de realizar aportes a la generación de estudios más elaborados, ya que gran parte de las investigaciones son realizada por personas que están a margen de las instituciones universitarias (Szmulewicz, 1998). Muchas de las investigaciones son el resultado de asesorías y estudios encargados por el sector público a equipos multidisciplinarios de profesionales. En este sentido, el sector público y el sector privado son quienes pueden utilizar la investigación generada en las universidades y sus escuelas de turismo, debido a que las decisiones que se tomen son mejores si se basan en estudios científicos (McIntosh y Gupta, 1993). En atención a este argumento el Servicio Nacional de Turismo (Sernatur), Chile, en su Política Nacional de Turismo señala:

...se incentivará a los centros universitarios del país a contribuir a un mejor conocimiento del comportamiento sectorial a través de investigación y tesis de grado (Sernatur, 1998, p. 13).

Por su parte, las empresas turísticas pueden entregar espacios a investigaciones de diferentes tópicos, como estudios operacionales, gerenciales, estratégicos etc. Estas pueden ayudar al mejoramiento de la actividad y a la generación de beneficios integrales para los empresarios y las áreas de desarrollo turístico, siempre y cuando los empresarios comprueben la utilidad de la investigación en su real dimensión y acción.

No debemos olvidar que el estudio del turismo no puede ser explicado desde una disciplina en particular, este requiere de un enfoque multidisciplinario (Garburns y Jafari, 1991), por tanto, son los centros universitarios con sus carreras y escuelas de turismo las que debiesen generar con mayor fuerza y actitud el fomento de la investigación turística. Ella requiere de un programa educativo coordinado entre instituciones públicas y privadas tanto en los niveles locales como nacionales, inclușo internacionales (Ansarah, 1998).

De lo anterior se desprende la utilidad de la investigación turística en todos 
los niveles, ella puede explicar, comprender y predecir el sistema, alimentando de forma sistemática la búsqueda de respuestas más elaboradas del turismo.

\section{Conclusión}

En consideración a la relación educación-investigación el "criterio ideal" se manifiesta como una señal para sensibilizarnos del estado presente de nuestra capacidad de creación e innovación para enfrentar el desarrollo del turismo, además es una invitación a trabajar en la optimización de las actuales estructuras de la educación turística. Un criterio ideal es una actitud de principio que podría permitir la construcción de conocimientos bien fundados del turismo y así fortalecer la disciplina, mejorar la actividad y consolidar a los profesionales del turismo, asícomo generar condiciones en los niveles abstractos (A), concreto educacional (CE) y concreto profesional (CP) en el proceso de formación del estudiante (Fig. 2).

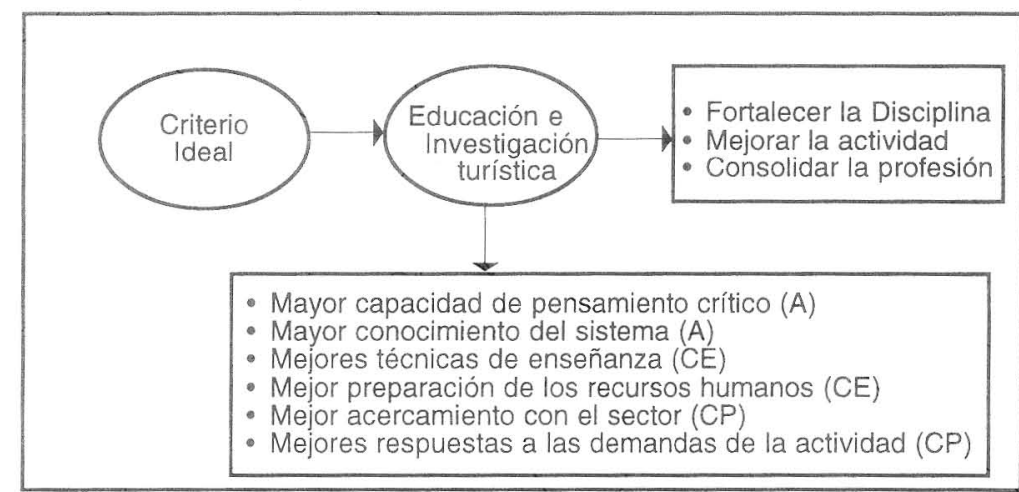

\section{FIGURA 2 - ESQUEMA DE LOS POSIBLES MEJORAMIENTOS DEL TURISMO} BASÁNDOSE EN EL CRITERIO IDEAL

En este contexto la enseñanza universitaria en turismo no sólo debe entregar buenos profesionales para desempeñarse en la actividad, sino que debe dar espacios al desarrollo del pensamiento crítico que nos conduzca a dar respuestas concretas sobre el turismo, en materia de su dimensión y alcance.
El criterio ideal aquí presentado es una reflexión para establecer aproximaciones más efectivas sobre la labor de la educación turística. A nuestro juicio lo importante es el proceso de análisis y crítica que puede surgir sobre los argumentos emitidos, ellos pueden dinamizar de alguna manera la búsqueda de nuevas perspectivas de estudios para el turismo a través de la educación e investigación. Creemos sin lugar a duda que nuevos enfoques sobre el tema nacen con propiedad en el seno de las universidades.

\section{Referencias Bibliográficas}

ANSARAH, M. 1998. Educación y formación del licenciado en turismo. Rute. n. 2. p. 55.68 BOULLON, R. 1988. Planeación del espacio furístico. México: Trillas

CARDENAS, F. 1986. Producto turistico. México: Trillas

DAMM, L. 1998. Educación profesional en Turismo. Gestion turistica, n. 2, p. 23-37.

GARBURN, J. Y JAFARI, J. 1991. Tourism Social Science. Anals of Tourism Research v. 18, n. 1.

JAFARI, J.1992. La cientifizacion del turismo. Clet, $n$. 15.

LICKONISH, L. 1994. Developing tourism destinations. México: Diana.

TEZ,D. IM L L formacio, la rehabilitació les noves modalitates turístiques. IIJORNADES DEGEOGRAFIA DEL PUR

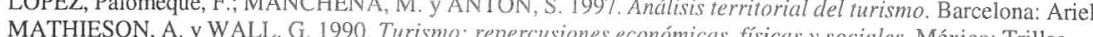
MCINTOSH R y GUPTA, S. 1993 Turismo: planeación a MOLINA, S. y RODRIGUEZ S. 1991. Plo Pfiction in Latinoamérica. México: Trilla

GANIZACIÓN MUNDIAL DEL TURISMOO. 1995. Educadores en turismo. España OMT. 1998. Introducción al turismo. España: OMT.

REJOWSKI, M. 1998. Investigación en turismo en las universidades Braileñas. Gestión Turística, n. 1, p. 46-61. SERVICIO NACIONAL DE TURISMO, 1998. Política Nacional de Turismo. Chile: Sernatur

P.MUlicz, P. 1998. Universidad e investigación turística en Chile. Turismo em Análise, São Paulo, v. 9 p. $107-121$.

YASIGI, E. 1998. Turismo uma esperanģa condiconal. São Paulo: Pleiade.

\section{Recibido en 15/9/00}

Aprobado en $27 / 10100$ 